\title{
Bloqueo analgésico femoral vs canal de aductores ecoguiado en artroplastia de rodilla, Ángeles Mocel
}

\section{Analgesic Ultrasound-guided adductor canal vs femoral block for knee arthroplasty at Ángeles Mocel}

\author{
Idaleyvis German-Córdoba ${ }^{1,2 *}$, José M. Athié-García ${ }^{3}$, José R. Villanueva-Solórzano ${ }^{4}$ y Mariela García-Bravo ${ }^{4}$ \\ ${ }^{1}$ Servicio de Anestesiología, Hospital Ángeles Mocel; ${ }^{2}$ Facultad Mexicana de Medicina, Universidad La Salle México; ${ }^{3}$ Dirección Médica, Hospital \\ Ángeles Mocel; ${ }^{4}$ Servicio de Anestesiología, Hospital ABC Observatorio. Ciudad de México, México
}

\begin{abstract}
Resumen
Introducción: La analgesia juega un papel importante en pacientes sometidos a cirugía de rodilla, por lo que el bloqueo femoral contra canal y los aductores guiados por ultrasonido son opciones que deben compararse para buscar la mejor analgesia. Objetivo: Determinar la seguridad y eficacia de bloqueo femoral contra canal de los aductores guiados por ultrasonido para analgesia en artroplastia de rodilla. Material y métodos: Se realizará un ensayo clínico aleatorizado simple ciego en pacientes operados de artroplastia de rodilla, en el Hospital Mocel, para comparar la eficacia en la analgesia del bloqueo femoral contra canal de los aductores. Se recolectaron las variables: edad, sexo, clasificación de la ASA (American Society of Anesthesiologists), tiempo de cirugía y efectos adversos, así como analgesia de rescate para su estudio. Resultados: Se obtuvo una muestra de 30 pacientes, divididos en dos grupos, para bloqueo femoral o del canal de los aductores guiados por ultrasonido, sin encontrarse diferencias significativas. Ambos grupos requirieron terapia de rescate de igual manera, no hubo diferencia en signos vitales y tampoco se reportaron efectos adversos en ninguno de los dos grupos. Conclusión: No existe diferencia significativa entre el bloqueo femoral y del canal de los aductores guiado por ultrasonido en pacientes sometidos a artroplastia de rodilla utilizando marcadores como la escala visual analógica y utilizando rescates de analgésicos.
\end{abstract}

Palabras clave: Bloqueo femoral. Canal de los aductores. Analgesia. Artroplastia de rodilla.

\begin{abstract}
Background: Analgesia plays an important role in patients undergoing knee arthroplasty, making ultrasound-guided femoral canal block and adductors; They are options that must be compared to find the best analgesia. Objective: To determine the safety and efficacy of femoral vs. adductor canal block ultrasound-guided for analgesia in knee arthroplasty. Material and methods: $A$ single-blind randomized clinical trial will be carried out in patients undergoing knee replacement at Hospital Mocel to compare the analgesic efficacy of femoral block against adductor canal. The variables of: age, sex, ASA, surgery time and adverse effects, as well as analgesia rescues were collected for the study. Results: A sample of 30 patients was obtained, divided into two groups, femoral blockade as adductor channel guided by ultrasound, without finding differences, both required rescue therapy in the same way, there was no difference in vital signs of both groups, thus Likewise, no adverse effects were reported in either group. Conclusion: There is no significant difference between ultrasound-guided femoral block and adductor canal in patients sometimes undergoing knee arthroplasty using markers such as an EVA scale and using analgesic rescues.
\end{abstract}

Key words: Femoral blockage. Adductor canal blockage. Analgesia. Knee arthroplasty.

\footnotetext{
Correspondencia:

Fecha de recepción: 21-07-2020

*Idaleyvis German-Córdoba

E-mail: idaleyvis@ hotmail.com

Fecha de aceptación: 13-04-2021

DOI: 10.24875/RHJM.200000901

Disponible en internet: 17-12-2021 Rev Hosp Jua Mex. 2021;88(4):175-180

www.revistahospitaljuarez.com 1405-9622/@ 2021 Sociedad Médico-Quirúrgica del Hospital Juárez de México, A.C. Publicado por Permanyer. Este es un artículo open access bajo la licencia CC BY-NC-ND (http://creativecommons.org/licenses/by-nc-nd/4.0/).
} 


\section{Introducción}

La articulación de la rodilla es una articulación sinovial, desde el punto de vista mecánico es troclear compuesta por dos articulaciones: la femorotibial, que es bicondílea, y la femororrotuliana, que es una tróclea'.

La amplia cápsula articular, recubierta de membrana sinovial, la hace susceptible al asiento de un importante número de afecciones sistémicas. Todo ello conlleva que sea la articulación de la rodilla en la que mayor número de enfermedades pueden presentarse $y$, por ende, en la que más comúnmente se realiza la artroplastia ${ }^{2}$.

La analgesia postoperatoria es fundamental para el ejercicio funcional precoz posterior a la cirugía de rodiIla. El bloqueo femoral (BF) ecoguiado ha sido aplicado en el manejo de la analgesia posterior a la artroplastia de rodilla, proporcionando manejo adecuado del dolor y por ende movilización precoz del paciente ${ }^{3}$. Se coloca al paciente en decúbito supino, se realiza palpación del pulso en el surco inguinal, se realiza previa asepsia y antisepsia y colocación de campos estériles y se coloca el transductor lineal de alta frecuencia movilizándolo hasta localizar la arteria femoral como un punto anecoico y lateralmente podemos ver un triángulo hiperecoico, indicando así que es el nervio femoral. Se inserta la aguja Stimuplex ${ }^{\circledR} 50 \mathrm{~mm}$ de lateral a medial al transductor (en plano) y se dirige hacia el nervio femoral. Una vez que localizamos la aguja adyacente al nervio realizamos aspiración cuidadosa previa y después colocación adecuada de $20 \mathrm{ml}$ de ropivacaína al $0.375 \%$, pudiendo localizar el signo de la «dona», que es cuando vemos que el nervio está completamente bañado de anestésico local. El grupo $\mathrm{F}$ corresponde al $\mathrm{BF}$, que se hará después de la aplicación del bloqueo neuroaxial, antes de iniciar la cirugía.

La cirugía de rodilla es un procedimiento quirúrgico que tiene como objetivo eliminar el dolor, reestablecer el movimiento de la articulación y la función a los músculos, ligamentos y otros tejidos blandos que la controlan, según la patología encontrada ${ }^{4}$.

Actualmente existen múltiples opciones analgésicas para la cirugía de rodilla; cada uno presenta sus ventajas y desventajas, por lo que no hay acuerdo acerca de un esquema único y superador. Es así como se describen a las opciones para tratamiento del dolor: la analgesia endovenosa sistémica, los bloqueos nerviosos (centrales y periféricos) y las técnicas infiltrativas ${ }^{5}$.
En este tipo de cirugía lo importante es la recuperación postoperatoria del paciente y para ello la contribución del anestesiólogo es de suma importancia, de ahí que una de las técnicas empleadas es el BF, que es muy adecuado para la cirugía en la cara anterior del muslo y para el manejo del dolor postoperatorio después de la cirugía de fémur y la rodilla $a^{6,7}$.

El principio de la técnica es abordar el nervio crural en el triángulo crural, inmediatamente por debajo del ligamento inguinal. El paciente es colocado en decúbito supino con la extremidad inferior en una abducción moderada $\left(10-20^{\circ}\right)$, la rodilla ligeramente flexionada y el pie en reposo ${ }^{8}$.

En general se puede realizar con la extremidad inferior en cualquier posición, siempre que se pueda palpar la arteria femoral y localizar el ligamento inguinal. El punto de punción se sitúa $1 \mathrm{~cm}$ por debajo del ligamento inguinal y externamente a la arteria femoral ${ }^{9}$.

Se inserta la aguja perpendicularmente a la piel o con una ligera inclinación proximal hasta que se producen las contracciones del cuádriceps. El paso a través de la fascia lata se percibe claramente, mientras que la expansión de la fascia ilíaca que rodea el nervio suele pasar desapercibida. Una vez en este punto se inyectan 20-30 ml del anestésico local elegido si se realiza una técnica de punción única. Numerosos estudios clínicos prospectivos confirman el uso de la analgesia continua mediante catéter situado en la vaina femoral en la cirugía de la rodilla ${ }^{10}$.

Las contraindicaciones son: alteraciones graves de la coagulación, alergia a anestésicos locales, infección cercana a la zona de punción, rechazo de la técnica por parte del paciente, enfermedades neurológicas o musculares, por las posibles repercusiones jurídico-legales ${ }^{11}$.

El bloqueo de canal aductor (BCA) está indicado para cirugía de rodilla, como lo son las artroscopias, reparación de ligamento cruzado anterior y reemplazo total articular, en donde el control adecuado del dolor y una pronta rehabilitación son básicos; este bloqueo es predominantemente un bloqueo nervioso de tipo sensitivo ${ }^{12}$. Es una opción para el manejo del dolor postoperatorio en procedimientos de rodilla, demostrando su eficacia analgésica, con una disminución en la escala visual analógica (EVA) durante las primeras 48 horas $^{13}$. Se coloca al paciente en decúbito supino, se realiza previa asepsia y antisepsia y colocación de campos estériles, se coloca la rodilla de la pierna en donde se realizará el bloqueo en arco y con el transductor lineal de alta frecuencia se rastrea la cara 
anterior del muslo del paciente a la altura del punto medio entre la ingle y el cóndilo medio, se identifica el fémur y movemos el transductor de manera lateral hasta encontrar el músculo sartorio, se introduce la aguja Stimuplex ${ }^{\circledR}$ de lateral a medial al transductor (en plano) y se dirige hacia el nervio sartorio, atravesándolo y llegando así al canal de los aductores, que se encuentra infrasartorial, ahí se realiza una aspiración cuidadosa y se administran los $10 \mathrm{ml}$ de ropivacaína al $0.375 \%$, asegurándonos de que el anestésico local diseque por debajo de la fascia del músculo sartorio, el grupo C, que corresponde al bloqueo del canal de los aductores se aplica después de la aplicación del bloqueo neuroaxial antes de iniciar la cirugía. Se considera una posible alternativa para el BF para proveer analgesia a la parte anteromedial de la rodilla y conservando la fuerza motora del músculo cuádriceps ${ }^{14}$.

Se ha visto en estudios recientes que el depósito de anestésico local guiado por ultrasonidos en el canal aductor sería útil para bloquear los nervios que atraviesan el canal y solo afectaría la rama motora del nervio vasto medial, conservando la función del resto de músculos del cuádriceps ${ }^{15}$.

López González efectuó en 2016 en España un estudio para evaluarla eficaciay seguridad del bloqueocanal aductor ecoguiado en pacientes programados para cirugía de artroplastia de rodilla en régimen ambulatorio en 20 pacientes con dos grupos de comparación: grupo BCA, a los que se les realizó un bloqueo del canal aductor ecoguiado con $20 \mathrm{ml} \mathrm{levobupivacaína} 0.5 \%$, previo a la cirugía; y grupo ID, a los que se les realizó una anestesia intradural con bupivacaína hiperbárica $0.5 \%$. En cuanto a los efectos secundarios no se encontraron diferencias significativas. La eficacia analgésica obtenida en ambos grupos fue similar, en el $95 \%$. No hubo retenciones de orina ni otras complicaciones asociadas a las técnicas. Solo hubo un caso de náuseas y vómitos en el grupo ID. Un total de 6 (60\%) pacientes precisaron tramadol de rescate en su domicilio durante las primeras 24 horas, uno pertenecía al grupo BCA y los otros $5(50 \%)$ al grupo ID $(p<0.05)^{16}$.

Jaeger realizó en 2013 un estudio prospectivo, aleatorizado y doble ciego en 11 voluntarios sanos en los que se comparaban dos bloqueos periféricosecoguiados (BCAvs. BF)ysurepercusiónenlafuerza delcuádriceps. Comparado con los valores basales, el bloqueo del canal aductor reduce la fuerza del cuádriceps un 8 vs. un $49 \%$ para el $\mathrm{BF}^{17}$.

Henshaw evaluó la analgesia posoperatoria en el bloqueo canal aductor ecoguiado en 155 pacientes operados de artroplastia de rodilla mediante dos grupos: $\mathrm{BCA}$ y bloqueo de compartimentos del psoas (PCB). Observaronque las puntuaciones de dolor fueron equivalentes a las 6 horas con descanso: BCA $1.0 \pm 2$ vs. PCB $1.1 \pm 2.2$, y con movimiento: BCA $1.6 \pm 2.6$ vs. PCB $1.5 \pm 2.8$. Adicionalmente, las puntuaciones de dolor en reposo y con movimiento a las 12,18 y 24 horas fueron equivalentes ${ }^{18}$.

En otro estudio, doble ciego, aleatorizado y controlado, seasignóaleatoriamentealos pacientes programados para artroplastia total de rodilla a dos grupos: uno utilizando el BCA ecoguiado y otro elBF. Fueronanalizados 48 pacientes para evaluar la fuerza muscular del cuádriceps y el rescate analgésico con morfina. Lafuerza del cuádriceps como porcentaje del valor inicial fue significativamente mayor en el grupo BCA en comparación con el grupo BF: (mediana [rango]) $52 \%$ [31-71] vs. 18\% [4-48], (intervalo de confianza del 95\%: 8-41; $p=0.004$ ). No hubo diferencias entre los grupos con respecto al consumo de morfina ( $p=0.94)$, dolor en reposo $(p=0.21)$, dolor durante la flexión de la rodilla $(p=0.16)$ o la fuerza muscular del aductor ( $p=0.39$ ); tampoco hubo una diferencia en los efectos adversos relacionados con la morfina o la capacidad de movilización $(p>0.05)^{19}$.

Kim evaluó el dolor postoperatorio en artroplastia de rodilla, en un grupo con $\mathrm{BCA}$ ecoguiado y en otro grupo con BF, además consumo de opioides a las 6 a 8, 24 y $48 \mathrm{~h}$ después de la administración de anestesia. Los pacientes recibieron un BCA o BF como componente de un esquema de analgesia multimodal. Cuarenta y seis pacientes recibieron $\mathrm{BCA} ; 47$ pacientes recibieron BF. A las 6 a $8 \mathrm{~h}$ postanestesia, con respecto a las puntuaciones de dolor de la escala de calificación numérica (1.0 [0.0-3.5] BCA frente a 0.0 [0.0-1.0] BF; $p$ $=0.019), 0$ al consumo de opioides (32.2 [22.4-47.5] BCA vs. 26.6 [19.6-49.0]; $p=0.0115$ ). A las 24 y $48 \mathrm{~h}$ después de la anestesia no hubo diferencias estadísticas significativas en los resultados de las puntuaciones de dolor o el uso de opioides entre los dos grupos $^{20}$.

\section{Material y métodos}

Este protocolo fue aprobado por el comité de ética e investigación del Hospital Ángeles Mocel, se realizó y firmó consentimiento informado de cada paciente. Es un ensayo clínico aleatorizado simple ciego mediante sorteo con método de tarjetas en dos grupos: C (canal de los aductores) y $\mathrm{F}$ (femoral), realizado en el Hospital Ángeles Mocel de la Ciudad de México, con duración de seis meses, en pacientes sometidos a artroplastia de rodilla manejando analgesia mediante $\mathrm{BF}$ y $\mathrm{BCA}$, ecoguiadas. 
El tamaño de muestra se establece con la fórmula para su estimación en estudios con diseño comparativo o ensayo clínico. Se hará la formación de dos grupos por método de muestreo aleatorizado simple, mediante un sorteo donde corresponderá a 15 fichas con letra $C$ y 15 con letra F. En ambos grupos aplicando ropivacaína $0.375 \%$, mediante BF y BCA ecoguiados, cuya efectividad se evaluará mediante la escala EVA al final de la cirugía, a las 2, 4, 6, 12 y 24 h; además se evaluarán los efectos hemodinámicos mediante monitoreo en el transquirúrgico cada media hora hasta el área de recuperación, otras variables del estudio serán: edad, sexo, clasificación de la ASA (American Society of Anesthesiologists), tiempo de cirugía y los efectos adversos, así como analgesia de rescate.

\section{Resultados}

Fueron seleccionados 30 pacientes para su análisis, operados de cirugía de rodilla, distribuidos en dos grupos: grupo $F(n=15) B F$ y grupo $C(n=15) B C A$ guiados por ultrasonido en ambas técnicas.

Del grupo $\mathrm{F}$ la edad media fue $67.9 \pm 8.7$ años, sexo femenino en $10(67 \%)$, artroplastia de rodilla derecha en 10 (67\%), ASA II en 7 (46.7\%). Del grupo C la edad media fue $69.2 \pm 10.5$ años, sexo femenino 11 (73\%), artroplastia de rodilla derecha con 8 (53.3\%), ASA II en $6(40.0 \%)$ (Tabla 1).

No se observaron efectos adversos y el rescate analgésico entre ambos grupos no presentó diferencias significativas, siendo de 5 (33\%) para cada uno, realizado con paracetamol principalmente, como se muestra en la figura 1.

El dolor postoperatorio medido con escala EVA se observó a las 2 h en escala 2 del grupo $F$ con 6 (40\%), del grupo $\mathrm{C}$ con 2 (13.3\%). A las $12 \mathrm{~h}$ en escala $1 \mathrm{el}$ grupo $\mathrm{F}$ con $4(26.7 \%)$ y el grupo $\mathrm{C}$ en $1(6.7 \%)$. A las $24 \mathrm{~h}$ en escala 5 el grupo $\mathrm{F}$ con $0 \%$ y el $\mathrm{C}$ en $1(6.7 \%)$. Ninguno fue significativo, como se detalla en la tabla 2.

De los resultados hemodinámicos se muestra la media de frecuencia cardiaca en latidos por minuto a los 30 en el grupo $F$ de $66.4 \pm 11$.2, en grupo $C$ con $74.7 \pm 12.9$. A los 90 en el grupo $F$ de $71.4 \pm 12.6$, en grupo $\mathrm{C}$ con $77.8 \pm 18.7$. Sin diferencias significativas.

El promedio de presión arterial sistólica medida en $\mathrm{mmHg}$ fue a los 60 minutos en el grupo $\mathrm{F}$ de $122.3 \pm$ 9.2 , en el grupo $C$ de $119.7 \pm 9.9$. A los 90 minutos en el grupo $F$ de $120 \pm 9.5$ y en el grupo $C$ de $117.6 \pm 12.6$. Sin diferencias significativas.

El promedio de presión arterial diastólica medida en $\mathrm{mmHg}$ fue a los 30 minutos en grupo $\mathrm{F}$ de $64.2 \pm 5.4$
Tabla 1. Características de los pacientes sometidos a artroscopia de rodilla en el Hospital Ángeles Mocel, por grupos de tipos de anestesia $(n=30)$

\begin{tabular}{|l|c|c|}
\hline Características & $\begin{array}{c}\text { Bloqueo } \\
\text { femoral } \\
(\mathbf{n}=15)(\%)\end{array}$ & $\begin{array}{c}\text { Canal de los } \\
\text { aductores } \\
(\mathbf{n}=15)(\%)\end{array}$ \\
\hline Edad & $67.9 \pm 8.7$ & $69.2 \pm 10.5$ \\
\hline Tiempo de cirugía en minutos & $87.7 \pm 22.0$ & $96.3 \pm 22.7$ \\
\hline $\begin{array}{l}\text { Sexo } \\
\text { Masculino }\end{array}$ & $5(33)$ & $4(27)$ \\
\hline Femenino & $10(67)$ & $11(73)$ \\
\hline $\begin{array}{l}\text { Tipo de cirugía } \\
\text { Artroplastia de rodilla derecha }\end{array}$ & $10(66.7)$ & $8(53.3)$ \\
\hline $\begin{array}{l}\text { Artroplastia de rodilla izquierda } \\
\text { Artroplastia total de rodilla } \\
\text { derecha }\end{array}$ & $4(26.7)$ & $7(46.7)$ \\
\hline ASA & $1(6.7)$ & 0 \\
\hline II & & \\
\hline III & $5(33.3)$ & $4(26.7)$ \\
\hline
\end{tabular}

ASA: American Society of Anesthesiologists.

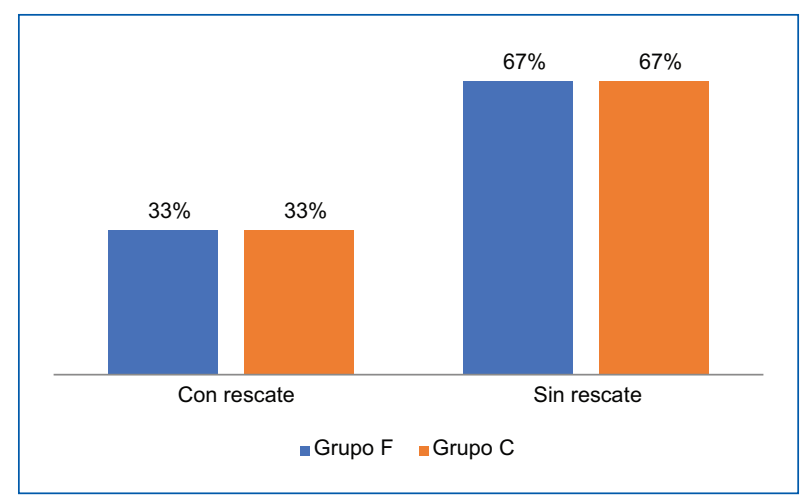

Figura 1. Rescate analgésico de los pacientes sometidos a artroscopia de rodilla en el Hospital Ángeles Mocel, por grupos de tipos de anestesia $(n=30)$.

y en el grupo $C$ de $64.8 \pm 5.2$. A los 60 minutos en el grupo $\mathrm{F}$ de $65.5 \pm 5.3$ y en el grupo $\mathrm{C}$ de $67.1 \pm 4.9$. Sin diferencias significativas.

La frecuencia respiratoria por minuto a los 30 en el grupo $F$ fue de $16.2 \pm 0.8$ y en el grupo $C$ de $16.5 \pm$ 1.3. A los 90 en el grupo $F$ de $17 \pm 1.0$ y en el grupo $C$ de $19.8 \pm 11.9$. Sin diferencias significativas.

La saturación de oxígeno se observó en promedio a los 30 minutos en el grupo $\mathrm{F}$ del $95.8 \% \pm 2.4$ y en el grupo $\mathrm{C}$ del $94.6 \% \pm 1.9$. A los 60 en el grupo $\mathrm{F}$ del $89.4 \% \pm 23.9$ y en grupo $C$ del $95.3 \% \pm 1.7$. Sin diferencias significativas. 
Tabla 2. Dolor postoperatorio, medido con escala visual analógica (EVA), de los pacientes sometidos a artroscopia de rodilla en el Hospital Ángeles Mocel, por grupos de tipos de anestesia $(n=30)$

\begin{tabular}{|c|c|c|c|}
\hline $\begin{array}{l}\text { Escala } \\
\text { EVA }\end{array}$ & $\begin{array}{l}\text { Bloqueo femoral } \\
(n=15)(\%)\end{array}$ & $\begin{array}{l}\text { Canal de los } \\
\text { aductores }(n=15)(\%)\end{array}$ & $\mathbf{p}^{*}$ \\
\hline $\begin{array}{l}2 \text { horas } \\
0 \\
1 \\
2 \\
3\end{array}$ & $\begin{array}{l}3(20.0) \\
3(20.0) \\
6(40.0) \\
3(20.0)\end{array}$ & $\begin{array}{l}5(33.3) \\
5(33.3) \\
2(13.3) \\
3(20.0)\end{array}$ & $<0.325$ \\
\hline $\begin{array}{l}4 \text { horas } \\
0 \\
1 \\
2 \\
3 \\
4 \\
5\end{array}$ & $\begin{array}{l}3(20.0) \\
2(13.3) \\
5(33.3) \\
3(20.0) \\
2(13.3) \\
0\end{array}$ & $\begin{array}{l}1(6.7) \\
8(53.3) \\
2(13.3) \\
2(13.3) \\
1(6.7) \\
1(6.7)\end{array}$ & $<0.624$ \\
\hline $\begin{array}{l}6 \text { horas } \\
1 \\
2 \\
3 \\
5 \\
6 \\
7 \\
8\end{array}$ & $\begin{array}{l}5(33.3) \\
3(20.0) \\
2(13.3) \\
2(13.3) \\
1(6.7) \\
1(6.7) \\
1(6.7)\end{array}$ & $\begin{array}{c}2(13.3) \\
9(60.0) \\
0 \\
3(20.0) \\
0 \\
1(6.7) \\
0\end{array}$ & $<0.935$ \\
\hline $\begin{array}{c}12 \text { horas } \\
1 \\
2 \\
3 \\
4 \\
5 \\
6 \\
7\end{array}$ & $\begin{array}{l}4(26.7) \\
5(33.3) \\
2(13.3) \\
1(6.7) \\
1(6.7) \\
1(6.7) \\
1(6.7)\end{array}$ & $\begin{array}{l}1(6.7) \\
9(60.0) \\
1(6.7) \\
1(6.7) \\
1(6.7) \\
1(6.7) \\
1(6.7)\end{array}$ & $<0.683$ \\
\hline $\begin{array}{c}24 \text { horas } \\
1 \\
2 \\
3 \\
4 \\
5\end{array}$ & $\begin{array}{c}2(13.3) \\
5(33.3) \\
6(40.0) \\
2(13.3) \\
0\end{array}$ & $\begin{array}{c}1(6.7) \\
7(46.7) \\
3(20.0) \\
3(20.0) \\
1(6.7)\end{array}$ & $<0.775$ \\
\hline
\end{tabular}

${ }^{*} p$, con U de Mann-Whitney.

\section{Discusión}

El objetivo de este estudio es comparar ambos bloqueos guiados por ultrasonido para observar si alguno de ellos tiene más eficacia para la analgesia postoperatoria en pacientes sometidos a cirugía de rodilla.

Como ya se comentó durante la introducción, existen otros autores que han comparado estos mismos bloqueos periféricos como técnica analgésica para cirugía de rodilla, teniendo resultados similares en todos, no se puede comprobar que alguno sea mas eficiente que otro en el control del dolor posoperatorio, y ambos grupos han requerido equiparable número de rescates analgésicos.
Una diferencia destacable en todos los estudios realizados es la selectividad sensitiva que tiene el BCA en comparación con el BF, el cual da bloqueo motor, por lo que el primero puede ser considerado una técnica de elección en pacientes que deben ser movilizados tempranamente.

Según la literatura y el estudio ya realizado, podemos asumir que no existen diferencias significativas para analgesia en pacientes sometidos a cirugía de rodilla con el BF y el canal de los aductores, siendo aún más recomendable este último por el mínimo bloqueo motor que tiene y capacidad mas temprana que tienen los pacientes para deambular. Sin embargo, no existen suficientes artículos que comparen la efectividad entre ambos abordajes, es necesaria una muestra mas amplia para poder realizar una mejor comparativa.

\section{Financiamiento}

Los autores no recibieron patrocinio para llevar a cabo este artículo.

\section{Conflicto de intereses}

Los autores declaran no tener conflicto de intereses alguno.

\section{Responsabilidades éticas}

Protección de personas y animales. Los autores declaran que para esta investigación no se han realizado experimentos en seres humanos ni en animales.

Confidencialidad de los datos. Los autores declaran que han seguido los protocolos de su centro de trabajo sobre la publicación de datos de pacientes.

Derecho a la privacidad y consentimiento informado. Los autores han obtenido el consentimiento informado de los pacientes y/o sujetos referidos en el artículo. Este documento obra en poder del autor de correspondencia.

\section{Bibliografía}

1. Flandry F, Hommel G. Normal anatomy and biomechanics of the knee Sports Med Arthrosc Rev. 2011;19(2):89-92.

2. Diaz López C. Uso de la artroplastia con fines terapéuticos. Ventajas y desventaja. Rev Clin Esp. 2003;200(1):46-58.

3. Choquet $O$, Zetlaoui PJ. Técnicas de anestesia regional del miembro inferior. EMC. 2015;41:1-24.

4. Choquet O, Capdevila X. Ultrasound-guided nerve blocks: the real position of the needle should be defined. Anesth Analg. 2012;114(5):929-30.

5. Saranteas T, Finlayson RJ, Tran DQ. Dose-finding methodology for peripheral nerve blocks. Reg Anesth Pain Med. 2014:39(6):550-5.

6. McCartney CJ, Patel S. Local anesthetic volume for peripheral nerve blocks: how low can (or should) we go? Reg Anesth Pain Med. 2012;37(3):239-41. 
Rev Hosp Jua Mex. 2021;88(4)

7. Zhai W, Wang X, Rong Y, Li M, Wang $\mathrm{H}$. Effects of a fixed low-dose ropivacaine with different volume and concentrations on interscalene brachial plexus block: a randomized controlled trial. BMC Anesthesiol. 2016;16(1):80.

8. Williams DJ, Walker JD. A nomogram for calculating the maximum dose of local anaesthetic. Anaesthesia. 2014;69:847-53.

9. Lirk P, Picardi S, Hollmann MW. Local anaesthetics: 10 essentials. Eur J Anaesthesiol. 2014;31:575-85.

10. Boezaart AP, editor. The anatomical foundations of regional anesthesia and acute pain medicine. Macroanatomy, microanatomy, sonoanatomy, functional anatomy. Bentham Books; 2016.

11. Gray AT. Atlas of ultrasound-guided regional anesthesia. Second Edition. Elsevier Saunders; 2013.

12. Chen J, Lesser J, Hadzic A, Reiss W, Resta-Flarer R. Adductor cana block can result in motor block of the quadriceps muscle. Reg Anesth Pain Med. 2014;39:170-1.

13. Torres Maldonado A. Bloqueo de canal aductor guiado por ultrasonido. Rev Mex Anestesiol. 2016;39:S249-S250.

14. Burckett-St D, Peng P, Girón L, Niazi A, Chan V, Agur A, et al. The nerves of the adductor canal and the innervation of the knee an anatomic study. Reg Anesth Pain Med. 2016;41:321-7.
15. Jaeger $\mathrm{P}$, Nielsen JK, Henningsen MH, Hilsted KL, Mathiesen O, Dahl JB. Adductor canal block vs. femoral nerve block and quadriceps strength. Anesthesiology. 2013;118:409-15.

16. López González JM, Jiménez Gómez BM, Areán González I, Padín Barreiro L, Illodo Miramontes G, López Álvarez S. Utilidad del bloqueo canal aductor ecoguiado en cirugía ambulatoria de artroplastia de rodilla. Cir May Amb. 2014;19(4):118-24.

17. Jaeger $P$, Nielsen JK, Henningsen MH, Hilsted KL, Mathiesen O, Dahl JB. Adductor canal block vs. femoral nerve block and quadriceps strength. Anesthesiology. 2013;118:409-15.

18. Henshaw DS, Jaffe JD, Reynolds JW, Dobson S, Russell GB, Weller RS An evaluation of ultrasound-guided adductor canal blockade for postoperative analgesia after medial unicondylar knee arthroplasty. Anesth Analg. 2016;122(4):1192-201.

19. Jæger P, Zaric D, Fomsgaard JS, Hilsted KL, Bjerregaard J, Gyrn J, et al. Adductor canal block vs. femoral nerve block for analgesia after total knee arthroplasty. regional anesthesia and pain medicine. 2013; 38(6):526-32

20. Kim DH, Lin Y, Goytizolo A, Kahn RL, Maalouf DB, Manohar A, et al. Adductor canal block vs. femoral nerve block for total knee arthroplasty. Anesthesiology. 2014;120:540-50. 